\section{Crop-health survey aims to fill data gaps}

The frequency and extent of crop losses caused by plant diseases and pests is another gap in our knowledge and understanding of agrifood systems (see P. Sukhdev et al. Nature 540, 33-34; 2016). This information is crucial for developing sustainable strategies to manage crop health.

Such losses can never be eliminated completely, and occur in many ecosystems. Declining crop health affects farmers, consumers and societies through higher input costs, lower outputs and damage to environmental and human health through the abuse and misuse of pesticides.

To try to quantify the problem, the International Society for Plant Pathology's online global survey of experts in crop health (https://globalcrophealth.org) is determining the importance of crop pests and diseases in five staple crops: wheat, rice, maize (corn), soya bean and potato. So far, around $75 \%$ of responses report losses every season, with one-third experiencing yield reductions of 5-60\% each year.

The survey, which ends on 31 January, has revealed large geographical gaps in the available expert knowledge on crop losses. Such gaps must be addressed to improve access to crop-health information and to increase understanding of today's needs and priorities for future sustainable food systems. Andy Nelson ${ }^{\star}$ University of Twente, the Netherlands. a.nelson@utwente.nl ${ }^{*}$ On behalf of 7 correspondents (see go.nature.com/2jtajyz for full list).

\section{Anthropocene: be wary of social impact}

As social scientists studying the work of the Anthropocene Working Group of the International Commission on Stratigraphy, we believe that the expertise of social scientists goes beyond developing a 'better' stratigraphic definition of the Anthropocene (E. Ellis et al. Nature 540, 192-193; 2016). Such knowledge should also be used to understand the likely consequences of any definition, particularly those given the weight of scientific credibility.

However it is defined, the Anthropocene could alter people's concepts of how humans interact with the natural world (see also N. Castree Nature 541, 289; 2017). Labels matter - a formal stratigraphic description might normalize human impacts on the planet and undermine efforts to minimize them, or lead people to ignore responsibilities for creating and managing the Anthropocene, which are unevenly spread around the world. Alternatively, it could inspire positive change and have a bigger impact on society than on stratigraphy. Social science can be used to develop concepts and a language for explaining the Anthropocene in both stratigraphic and political terms. George Holmes University of Leeds, UK.

Jacob Barber University of Edinburgh, UK.

Johannes Lundershausen University of Tübingen, Germany. g.holmes@leeds.ac.uk

\section{Anthropocene: keep communication clear}

Regarding definitions of an Anthropocene epoch, we disagree with Erle Ellis and colleagues' contention that "Earth sciences long ago moved away from defining precise stratigraphic boundaries to developing records of continuous change" (Nature 540, 192-193; 2016).

Precise boundaries are the basis for defining geological time, a prerequisite for the correlation of abiotic and biotic events and the understanding of the rates and timing of biological and geological processes on our planet. Earth sciences, through the International Commission on Stratigraphy of the International
Union of Geological Sciences, continue to this day to define precise global boundaries, which in turn allows scientists to communicate with each other and with the public alike.

Developing records of change (continuous, discontinuous or one-time-only) in the study of Earth's history neither competes with nor detracts from scientists' key contribution in providing tools for unambiguous communication.

Lucy E. Edwards US Geological Survey, Reston, Virginia, USA.

David A. T. Harper Durham University, UK.

Philip L. Gibbard University of Cambridge, UK.

leedward@usgs.gov

\section{Detecting particles of dark matter}

Your article on the Axion Dark Matter eXperiment (ADMX) suggests that the lattice quantum chromodynamics (QCD) calculation by S. Borsanyi et al. (Nature 539, 69-71; 2016) might be bad news for the ADMX because it could place the axion mass beyond the detector's reach (see Nature http://doi.org/bxf8; 2016). We find this inference misleading.

The axion is a very wellmotivated hypothetical particle because it solves a puzzle in the standard model of elementary particles (the 'strong CP problem'; see J. E. Kim and G. Carosi Rev. Mod. Phys. 82, 557; 2010) and because a cold population of axions is naturally produced in the early Universe that may constitute dark matter today.

The properties of the axion depend for the most part on a single parameter that may be taken as the axion mass. Unfortunately, the mass is poorly constrained and might plausibly range from $10^{-6}$ to $10^{3}$ microelectronvolts. Inconclusive theoretical arguments have been put forth in support of each part of that range.

The only way to find out the axion mass is through experimental observation. Axion dark-matter detection methods are emerging that, in principle, cover the whole mass range. Experiments that use the resonant cavity technique, such as ADMX, are capable of discovering axions today. They should be vigorously pursued. The aforementioned lattice QCD calculation does not change this fundamental picture.

Jihn E. Kim Seoul National University, Seoul, South Korea. Pierre Sikivie University of Florida, Gainesville, USA.

Steven Weinberg The University of Texas at Austin, USA. sikivie@phys.ufl.edu

\section{Progressive taxes for sustainability}

One way to achieve a more sustainable society would be to impose progressive taxes on goods that are particularly detrimental to the environment when consumed. Marginal tax rates would increase with an individual's consumption of those goods - for example, a person's annual flight history could be used to compute the fee for his or her next ticket.

Fixed-rate consumption taxes present equity issues, which hinder their applicability and effectiveness by placing much of the burden on those with low incomes (see, for example, C. A. Grainger and C. D. Kolstad Environ. Resour. Econ. 46, 359-376; 2010). Implementing progressive consumption taxes on specific goods is now possible because of the widespread use of smartphones, credit cards and the Internet, which mean that we can keep track of individual consumption patterns.

Although such taxes may be perceived as a limitation on our freedom, in my view they would be a credible implementation of the 'polluter pays' principle. Francesco Orsi Kansas State University, Manhattan, USA. forsi@ksu.edu 